\title{
A METHOD FOR DERIVING THE MEAN VOLUME SCATTERING PHASE FUNCTION FOR ZODIACAL DUST
}

\author{
S. S. Hong \\ Space Astronomy Laboratory \\ University of Florida \\ $1810 \mathrm{NW}$ 6th Street \\ Gainesville, Florida 32609 \\ USA
}

ABSTRACT. A linear combination of 3 Henyey-Greenstein phase functions is substituted for the mean volume scattering phase function in the zodiacal light brightness integral. Results of the integral are then compared with the observed brightness to form residuals. Minimization of the residuals provides us with the best combination of HenyeyGreenstein functions for the scattering phase function of zodiacal dust particles.

\section{INTRODUCTION}

Most theoretical methods for deriving an empirical scattering function for interplanetary dust particles require taking derivatives of the observed zodiacal light brightness. Observational errors are easily amplified in the process of differentiation and likely to distort the scattering function derived therefrom. As an alternative, we take an integral approach: A utilization of the versatile Henyey-Greenstein (HG) function as a parameterized trial scattering function will enable us to employ the method of non-linear least, squares in determining the mean volume scattering phase function for zodiacal dust particles.

\section{BRIGHTNESS INTEGRAL WITH THE HENYEY-GREENSTEIN FUNCTION}

A linear combination of three HG functions

$$
\phi\left(\theta ; g_{k}, w_{k}\right)=\sum_{k=1}^{3} \frac{w_{k}}{4 \pi} \frac{\left(1-g_{k}^{2}\right)}{\left(1+g_{k}^{2}-2 g_{k} \cos \theta\right)^{3} / 2}
$$

having different asymmetry factors, $g_{k}$, adequately describes such general features as the forward peak, isotropic middle, and backward enhancement in a particle scattering pattern. Here, $w_{k}$ represents relative weights of the three components, and $\theta$ denotes scattering angle. With the usual power-1aw relation, $n(r)=n(r / r)$, for the heliocentric density distribution of particles, the zodiacal light 215

R. H. Giese and P. Lamy (eds.), Properties and Interactions of Interplanetary Dust, 215-218.

(C) 1985 by D. Reidel Publishing Company. 
brightness $Z(\varepsilon)$ in the ecliptic is given by

$$
Z(\varepsilon)=\frac{\zeta \bar{\sigma}}{\sin ^{\nu+1}{ }_{\varepsilon}} \int_{\varepsilon}^{\pi} \Phi(\theta) \sin ^{\nu} \theta \mathrm{d} \theta,
$$

where $\zeta=F_{o} n_{o} r_{0}$ with $F_{o}$ being the solar flux at $r=r_{o} ; \Phi(\theta)$ is the mean volume scattering phase function of interplanetary particles and $\bar{\sigma} \Phi(\theta)$ becomes the differential scattering cross-section. For an arbitrary $v$, one may rely on numerical means to evaluate the brightness integral. However, for $\nu=1$, the integral with HG scattering phase function has an analytical solution. Additionally, one can generalize the result of the special case $v=1$ to cases other than unity.

For $v=1$, substitution of eqn (1) into eqn (2) for $\Phi(\theta)$ gives

where

$$
\mathrm{Z}(\varepsilon)=\frac{\zeta_{1} \bar{\sigma}_{1}}{4 \pi} \sum_{\mathrm{k}=1}^{3} \mathrm{w}_{\mathrm{k}} \mathrm{H}\left(\varepsilon ; g_{\mathrm{k}}\right) \text {, }
$$

$$
\mathrm{H}\left(\varepsilon ; \mathrm{g}_{\mathrm{k}}\right)=\frac{1}{\sin ^{2} \varepsilon} \frac{1-\mathrm{g}_{\mathrm{k}}}{\mathrm{g}_{\mathrm{k}}}\left[\frac{1+\mathrm{g}_{\mathrm{k}}}{\left(1+\mathrm{g}_{\mathrm{k}}^{2}-2 \mathrm{~g}_{\mathrm{k}} \cos \varepsilon\right)^{\frac{1}{2}}}-1\right]
$$

for non-zero asymmetry factors and $H(\varepsilon ; 0)=1 /(1-\cos \varepsilon)$ for zero asymetry factor; $H\left(\pi ; g_{k}\right)=\frac{1}{2}\left(1-g_{k}\right) /\left(1+g_{k}\right)^{2}$ at the anti-solar point. Notice that subscript " 1 " is attached to $\zeta$ and $\bar{\sigma}$ to remind us that $\nu=1$.

Normalizing both theoretical $\mathrm{Z}(\varepsilon)$ and observed $\mathrm{Z}_{\text {obs }}(\varepsilon)$ brightness to their corresponding values at elongation $90^{\circ}[z(\varepsilon) \equiv Z(\varepsilon) / Z(\pi / 2)$ and $\left.\mathrm{z}_{\text {obs }}(\varepsilon) \equiv \mathrm{Z}_{\text {obs }}(\varepsilon) / \mathrm{Z}_{\text {obs }}(\pi / 2)\right]$, we form the reduced chi-square as

$$
x_{r}^{2}=\frac{1}{i m x-p} \sum_{i=1}^{i m x} n^{2}\left(\varepsilon_{i}\right)\left[z\left(\varepsilon_{i}\right)-z_{o b s}\left(\varepsilon_{i}\right)\right]^{2},
$$

where imx is the number of observational values used in the analysis and $p$ is the number of parameters used in the parametric representation of the unknown scattering function. To make the importance of each observed brightness nearly equal, we introduce the statistical weighting function $n^{2}\left(\varepsilon_{i}\right)=1 / z_{\text {obs }}\left(\varepsilon_{j}\right)$. Minimization of the reduced chi-square with respect to the HG parameters determines the best parameters for the mean volume scattering phase function $\Phi_{1}(\theta)$ of the zodiacal dust, the subscript " 1 " again serving as a reminder of $\nu=1$.

Since the equation of direct differential inversion

$$
\Phi(\theta=\varepsilon)=\frac{1}{\zeta \bar{\sigma}}\left[-(\nu+1) \cos \varepsilon Z(\varepsilon)-\sin \varepsilon \frac{\partial}{\partial \varepsilon} Z(\varepsilon)\right]
$$

holds for an arbitrary power-law exponent (Dumont, 1976), our special case result gives

$$
\Phi_{1}(\theta=\varepsilon)=\frac{1}{\zeta_{1} \bar{\sigma}_{1}}\left[-2 \cos \varepsilon Z(\varepsilon)-\sin \varepsilon \frac{\partial}{\partial \varepsilon} Z(\varepsilon)\right] .
$$

Therefore we can eliminate the differential term from eqn (6) to obtain for arbitrary $v$ : 


$$
\Phi(\Theta=\varepsilon)=\frac{\zeta_{1} \bar{\sigma}_{1}}{\bar{\sigma}_{\zeta}}\left[\Phi_{1}(\varepsilon)-(\nu-1) \cos \varepsilon \frac{Z(\varepsilon)}{\zeta_{1} \bar{\sigma}_{1}}\right] .
$$

Once $\nu$ is fixed, all the quantities inside the square brackets become known; hence the functional form of $\Phi(\theta)$ is determined. Since generalization by eqn (8) does not involve any differentiation, the influence of observational errors on the derived scattering function becomes minimal.

\section{THE MEAN VOLUME SCATTERING PHASE FUNCTION}

Non-linear least squares analysis given to combined ground and space observations (Dumont and Sánchez, 1975; Leinert, Link, Pitz and Giese, 1976) provides us with the solution in Table $I$. Although $w_{3}$ is as small as 0.005 , the third HG component plays an increasingly important role towards large scattering angles. For example, $28 \%$ of $\Phi_{1}(\pi)$ comes from this component, while only $7 \%$ comes from the more heavify weighted first component. Observed brightening of the zodiacal light towards the anti-solar point requires this strongly backward throwing $\left(\mathrm{g}_{3}=\right.$ -0.813) HG function in the mean volume scattering function of the zodiacal dust. The resulting scattering phase function is depicted in Fig 1 by a dashed line. In the same figure, the zodiacal light brightness (solid line) resulting from eqn (3) with the parameter values from Table $I$ is compared with the observations (dots) at 23(=imx) selected elongations.

Table I. Henyey-Greenstein Parameters for $\Phi_{1}(\theta)$

\begin{tabular}{lccr}
\hline Asymmetry factors $\mathrm{g}_{\mathrm{k}}$ & 0.698 & -0.203 & -0.813 \\
Relative weights $\mathrm{w}_{\mathrm{k}}$ & 0.665 & 0.330 & 0.005 \\
$\zeta_{1} \bar{\sigma}_{1}$ in $\mathrm{s}_{10}(\mathrm{~V})_{\odot} \cdot \mathrm{sr}$ & & $4.61 \times 10^{3}$ & \\
\hline
\end{tabular}

The uniqueness of the solution given in Table I can be assessed by examining whether or not the equal chi-square contours have an isolated minimum in the HG parameter planes. We first kept one asymmetry factor fixed, then at every possible grid point of the remaining two asymmetry factors, we found the local-minimum reduced chi-square by varying the relative weights. As an example, we displayed in Fig 2 such contours on the $\left(g_{1}, g_{2}\right)$ plane with $g_{3}$ and $w_{3}$ kept at their best values 4 To each contour level chi-square values are given in units of $10^{-4}$; a few percent random error in the observation corresponds to roughly 20 in this unit. The contours in Fig 2 clearly bear out a well-defined minimum, and the same was found from the contours on both $\left(g_{2}, g_{3}\right)$ and $\left(g_{3}, g_{1}\right)$ planes.

$\Phi_{1}{ }^{1}(\theta)$ was generalized by eqn (8), and the results are displayed in Fig 3. At low scattering angles the derived scattering function is sensitive to the power-law exponent; for $v>1$, it reverses its rise towards the forward direction. As observations below elongation $15^{\circ}$ were excluded from the analysis, the reality of such reversal and its relation to the density very close to sun needs additional investigation. 

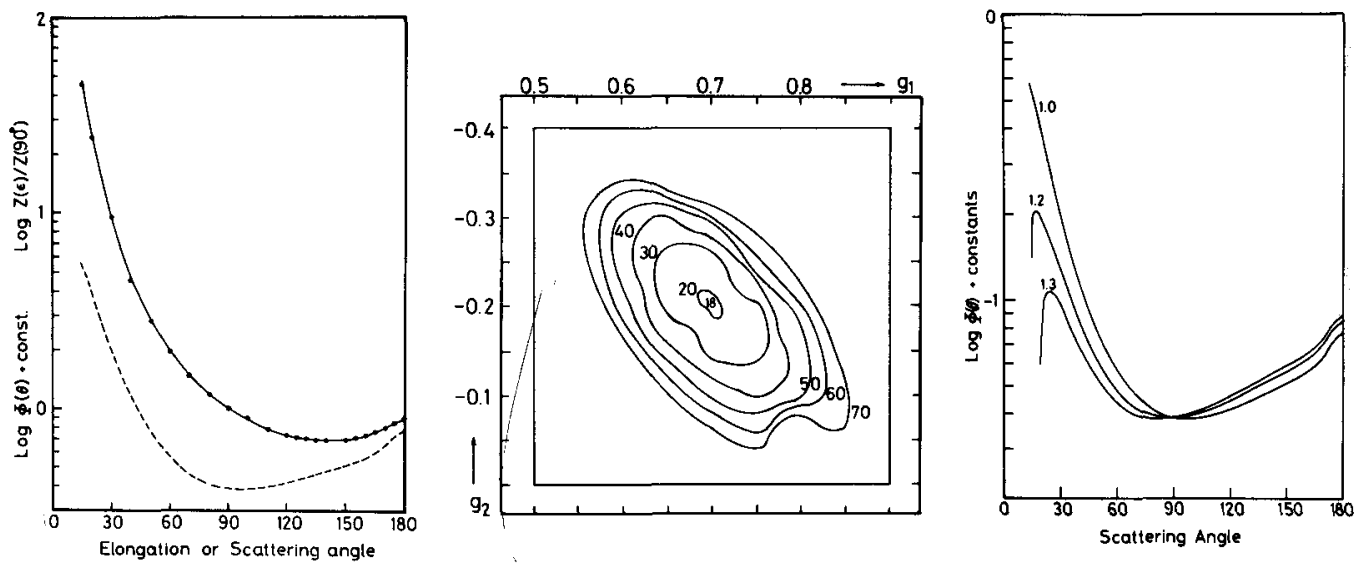

Fig 1: Comparison of observational (dots) and computed (solid line) brightness of zodiacal light using the dashed line HG phase function. Fig 2: Projection of minimum chi-square surface on the $\left(g_{1}, g_{2}\right)$ plane. Fig 3: Generalization of $\Phi_{1}(\theta)$ to cases $\nu$ slightly larger than unity.

\section{CONCLUDING REMARKS}

Versatility of the HG function in portraying widely varying scattering patterns and its rendering of an analytical solution to the brightness integral enables us to establish the uniqueness of the derived solution. The overall scattering characteristics found from our integral approach are in general agreement with previous investigations. What is unique about this study is that characteristics of the mean volume scattering phase function for the zodiacal dust are quantified by specifying numerical values for the HG parameters. Finally, it should be pointed out that an extension of this method to polarization amounts to only changes in notation, because the brightness integral holds for both components of the polarized brightness.

ACKNOWLEDGEMENT: This work was supported by the U.S. Air Force Office of Scientific Research.

\section{REFERENCES}

Dumont, R., Sánchez, F.: 1975, Astron. Astrophys. 38, 405. Dumont, R.: 1976, in Interplanetary Dust and Zodiac高 Light, Lecture Notes in Physics 48, ed. by H. Elsässer and H. Fechtig, p. 115. Leinert, C., Link, H., Pitz, E., Giese, R. H.: 1976, Astron, Astrophys. $\underline{\underline{47}}, 221$. 